\title{
Zu den römischen Inschriften aus Mürlenbach in der Vulkaneifel (Gallia Belgica)
}

\author{
Krešimir Matijević
}

Aus Mürlenbach in der Vulkaneifel, Verbandsgemeinde Gerolstein (Rheinland-Pfalz), sind verschiedene römische Funde und Befunde bekannt. ${ }^{1}$ Die Ortsgemeinde liegt 4-5 km östlich der römischen Fernstraße Trier - Bitburg - Köln, von der einige Kilometer nördlich von Bitburg eine Querstraße in die Vulkaneifel hinein nach Mürlenbach führt. ${ }^{2}$ Im Sommer 1841 ist an einer nicht mehr genauer zu lokalisierenden Stelle ein Tempel gefunden worden. ${ }^{3}$ Laut FRANZ CHASSOT VON FLORENCOURT lag er eine halbe Stunde östlich von Mürlenbach, ,auf einer mittlern Abstufung des die linke Thalwand der Kyll bildenden Höhenzuges [... im] District ,ober dem Hasselsseifen“" “. ${ }^{4}$ JAKOB SCHNEIDER schreibt, dass sich auf der gegenüberliegenden Talwand die Burg Mürlenbach, auch Bertrada-Burg genannt (s.u.), befindet. ${ }^{5}$ Der Tempel sei etwa $10 \mathrm{~m}$ lang und etwas weniger breit gewesen. ${ }^{6}$ FELIX HETTNER hat auf Grundlage dieser Größe vermutet, dass es sich um einen Umgangstempel gehandelt haben könnte und dass die Cella bei der privaten Ausgrabung übersehen worden ist. ${ }^{7}$ Einige wenige Münzen, die beim 1841 entdeckten Heiligtum aufgefunden wurden, deuten darauf hin, dass es noch im 4. Jahrhundert genutzt wurde. ${ }^{8}$

Direkt bei der Entdeckung des Tempels ist in den Trümmern desselben auch eine römische Votivinschrift für Deus Caprio zum Vorschein gekommen, die ein Mann namens Lucius Teđđiatius Primus zu Ehren des vergöttlichten Kaiserhauses gestiftet hat. ${ }^{9}$ Es handelt sich um eine Kalksteintafel, die am Tempel angebracht gewesen sein wird. Die Maße betragen 31 x noch $52 \times 11 \mathrm{~cm} .{ }^{10}$ Buchstabenhöhe: $4-5 \mathrm{~cm} .{ }^{11} \mathrm{Zu}$ lesen ist (nach Photographie ${ }^{12}$ ):

\footnotetext{
${ }^{1}$ Siehe den Fundstellenkatalog bei KRAUSSE 2006, $165 f$.

${ }^{2}$ Siehe HAGEN ${ }^{2} 1931,163$.

${ }^{3}$ Siehe zu dem Heiligtum FlorenCOURT 1842, 53-58; WellensteIn in: Bonner Jahrbücher 14, 1849, 176f.; AUS'M WEERTH 1876, 57; HETTNER 1911, 54; WACKENRODER 1927, 416; HAGEN 21931, 164; GRENIER 1960, 898; HoRne/KING 1980, 438; CABUY 1991, 361f.; HenRICH 2006, 34, 65f., 173; KRAUSSE 2006, 166 Nr. 1209; GHETTA 2008, 324.

${ }^{4}$ FLORENCOURT 1842, 53 (z.T. unter Berufung auf WELLENSTEIN). WELLENSTEIN in: Bonner Jahrbücher 14, 1849, 176 gibt wiederum, wohl irrtümlich, das Fundjahr 1840 und den Namen des Distrikts als „Haasen-Seifen“ an; siehe auch AUS’M WEERTH 1876, 57 („,,ober dem Haffelsseifen““ - wohl ein Druckfehler); WACKENRODER 1927, 416: „,,ober dem Hasselseifen“ (auch Haasenseifen)“; MÜLLER 1932, 44 : „,auf der Gemarkung Hasselseifen“; HENRICH 2006, 34 und GHETTA 2008, 324: „,Haasensiefen““. Fundjahr 1840 auch bei MÜLLER 1932, 44 und KRAUSSE 2006, 166.

${ }^{5}$ Siehe SCHNEIDER 1843, 83.

${ }^{6}$ Siehe FLORENCOURT 1842, 53.

${ }^{7}$ Siehe HeTtNer 1911, 54. Anders herum HoRnE/KING 1980, 438, die in den entdeckten Mauern die Cella vermuten und den Umgang für nicht entdeckt halten.

${ }^{8}$ Siehe HENRICH 2006, 173; GHETTA 2008, 324.

${ }^{9}$ CIL XIII 4142; zuletzt zu dieser Inschrift WeILER/REINHARD 2018, 192-194 Nr. 87.

${ }^{10}$ Nach BinsfelD u.a. 1988, 28 Nr. 41.

${ }^{11}$ Nach HeTtNer 1893, 66 Nr. 113.

${ }^{12}$ Bei Binsfeld u.a. 1988, Abb. 41 auf Taf. 11.
} 


\author{
In ${ }^{\circ} h$ (onorem) $\left[^{\circ}\right] d$ (omus) ${ }^{\circ}$ de(ivinae) $\mathrm{Zu}$ Ehren des vergöttlichten Kaiserhauses \\ Deo ${ }^{\circ}$ Caprion [i] \\ L(ucius) ${ }^{\circ}$ Teđdiatius \\ (hat) für den Gott Caprio \\ Lucius Teddiatius \\ Primus. \\ Primus (diesen Tempel geweiht).
}

An der unteren und linken Seite sind noch Teile eines profilierten Rahmens erkennbar. Links von der zweiten Inschriftzeile ist zudem eine Muschel in den Stein gearbeitet. Vielleicht hat der Rahmen an dieser Stelle eine Aussparung in Form einer Ansa aufgewiesen. Dem Namen nach handelt es sich um einen Einheimischen, der durch seine Tria Nomina eine gehobene Rechtsstellung anzeigt. ${ }^{13}$ Wahrscheinlich datiert die Weihung in die 2. Hälfte des 2. Jahrhunderts n.Chr. ${ }^{14}$

THOMAS LOBÜSCHER hat 1997 auf eine offensichtlich identisch lautende, nur literarisch überlieferte und inzwischen zerstörte Inschrift hingewiesen, bei der es sich um einen Altar gehandelt haben soll. ${ }^{15}$ Dieser Titulus ist zwar in die Année Épigraphique aufgenommen, ${ }^{16}$ in der Forschung aber noch nicht als eigenständiges Dokument anerkannt worden, was daran liegen dürfte, dass er in den epigraphischen Datenbanken mit dem Altfund identifiziert wird. ${ }^{17}$

LOBÜSCHER bezog seine Information aus der „Heimatgeschichte von Mürlenbach“, die 1932 von JOHANNES HUBERT MÜLlER publiziert wurde. Bei diesem Mann handelt es sich um einen im Jahre 1876 geborenen Telegrafenwerkführer, der in seiner Ortsgemeinde Mürlenbach verschiedentlich öffentlich hervorgetreten ist. ${ }^{18}$

MÜLLER gibt die Maße 120 x 90 x $90 \mathrm{~cm}$ und berichtet, es habe sich um ein Denkmal aus Sandstein gehandelt. ${ }^{19}$ Es sei „im Jahre 1896 beim Bau einer Scheune an meinem elterlichen Hause von mir freigelegt [worden ...]. Ich hatte die Absicht, den Stein dem Provinzialmuseum in Trier zur Verfügung zu stellen, doch wurde derselbe von einem Maurer flegelhafterweise vernichtet. “20 Folgende Lesung ist bei MÜLLER abgedruckt:

\author{
IN H (ONOREM) \\ D (IVINAE) CAPRIONI \\ L(UCIUS) TEDDIATIUS
}

\footnotetext{
${ }^{13}$ Siehe KaKOSCHKE 2010, 170 GN 497.

${ }^{14}$ Darauf deutet zum einen die Einleitung INHDD und zum anderen die Voranstellung von Deus hin. Da der Mann ein Praenomen angibt, ist eine Datierung noch ins 2. Jahrhundert wahrscheinlicher als eine in die 1. Hälfte des 3. Jahrhunderts, wie sie LOBÜSCHER 1997, 160 und WEILER/REINARD 2018, 192 vertreten.

15 Siehe LOBÜSCHER 1997, 159-161.

${ }^{16}$ AE 1997, 1144.

${ }^{17}$ EDCS-1060111 listet AE 1997, 1144 lediglich als zusätzlichen Nachweis für CIL XIII 4142 auf (letzter Zugriff am 27.01.2021); der EDCS folgen WEILER/REINHARD 2018, 192-194 Nr. 87. EDH-HD049698 geht ähnlich vor, sieht AE 1997, 1144 aber offensichtlich als Korrektur von CIL XIII 4142 an, da nur die Lesung der literarisch überlieferten Inschrift geboten wird (letzter Zugriff am 27.01.2021).

${ }^{18}$ Siehe die „Datenbank der Kulturgüter in der Region Trier“ zum von Müller erbauten MuttergottesKapellchen in Hanert, Gemeinde Mürlenbach (https://kulturdb.de/einobjekt.php?id=43991; letzter Zugriff am 27.01.2021).

${ }^{19}$ Siehe MÜLLER 1932, 44

${ }^{20}$ Ebd.
} 
Mit LOBÜSCHER kann man die Lesung verbessern zu: ${ }^{21}$

\author{
In h(onorem) [d(omus) d(ivinae)] \\ $D$ [eo] Caprioni \\ L(ucius) Teddiatius \\ [Primus] \\ $------?$
}

Weiterer Text könnte im unteren Bereich der Inschrift gefolgt sein. Sicher ist jedenfalls, dass es sich um eine zweite Dedikation desselben Mannes gehandelt hat, die in etwa zur selben Zeit wie die oben erwähnte dargebracht wurde.

Schon 1832 seien (offensichtlich bei MüLLERs Elternhaus), ,in dem Ortsteil ,Op der Stroaß ${ }^{‘}$ die Fundamente eines römischen Tempels freigelegt [worden], der dem Gotte Caprio, dem Gotte der Hirten, geweiht war.“22 Letzteres wird augenscheinlich aus der später von MÜLLER am selben Ort entdeckten Inschrift geschlossen. Da MÜLLER direkt im Anschluss von dem in der Forschung bekannten Tempel ,,auf der Gemarkung Hasselseifen“ und der früher entdeckten Inschrift berichtet, ${ }^{23}$ scheint es sich bei dem von ihm erwähnten Heiligtum um ein weiteres zu handeln. Allerdings gibt MÜLLER als Größe „ungefähr $30 \mathrm{Fuß} \mathrm{im} \mathrm{Geviert“} \mathrm{an,}{ }^{24}$ welche dem in der Forschung bekannten Tempel entspricht, für den er wiederum keine Größenangaben macht. ${ }^{25}$

MÜLLER liefert in seinem Büchlein verschiedene Details und geht auch auf den über 50 Jahre zuvor entdeckten Altfund ein, was den Eindruck der Zuverlässigkeit hervorruft. Anders als LOBÜSCHER angibt, identifiziert MÜLLER das Denkmal als „Altarsockel“. ${ }^{26}$ In der Tat könnten die Maße für einen Statuensockel sprechen, zumal MÜLLER an gleicher Stelle vermeldet, dass ,im Jahre 1928 [...] beim Umbau meines elterlichen Hauses der Kopf einer Figur mit schönen Zügen gefunden [wurde], wahrscheinlich der Kopf des Caprio, der auch wieder an gut sichtbarer Stelle eingemauert wurde.“27 Im selben Jahr sei 60 m entfernt im „Nachbarhause von Anton Marxen bei Umbauarbeiten ein mit Blumenranken versehenes Säulenstück aus Sandstein gefunden“"worden, worin MÜLLER ebenfalls ein Element des Tempels vermutet. ${ }^{28}$

Deus Caprio ist bislang nur aus Mürlenbach bekannt, so dass es sich vielleicht um eine lokale Gottheit handelt. ${ }^{29}$ SASCHA WEILER und PATRICK REINARD denken wegen der muschelförmigen Verzierung an der Inschrifttafel, dass Caprio als Wasserbzw. Heilgott charakterisiert werden könnte und verweisen auf eine naheliegende Quelle bei Mürlenbach. ${ }^{30}$ Tatsächlich ist die Entfernung zur Quelle unbekannt, weil der hier besprochene Tempel nicht mehr genau zu lokalisieren ist (s.o.). Zudem ist nahe der

\footnotetext{
${ }^{21}$ LOBÜSCHER ergänzt das Cognomen des Mannes am Ende der dritten Zeile; hier wird die Nennung in der vierten Zeile, wie in der erhaltenen Inschrift, vertreten.

22 MÜLLER 1932, 44.

${ }^{23} \mathrm{Ebd}$.

${ }^{24}$ MÜLLER 1932, 44.

${ }^{25}$ Im Fundstellenkatalog KRAUSSE 2006, 165f. findet sich kein Hinweis auf das 1832 entdeckte Heiligtum.

${ }^{26}$ MÜLLER 1932, 44.

${ }^{27}$ Ebd.

${ }^{28}$ Ebd.

${ }^{29}$ Siehe LOBÜSCHER 1997, 159-161 (mit weiteren Beispielen); HENRICH 2006, 65 f.

${ }^{30}$ WEILER/REINARD 2018, 193. Siehe auch GRENIER 1960, 898, der das Heiligtum unter den ,sanctuaires de l'eau“ aufführt.
} 
Quelle bereits ein Befund überliefert, bei dem es sich wahrscheinlich um einen weiteren Tempel handelt. ${ }^{31}$

Ebenfalls aus Mürlenbach stammt das Fragment einer Grabinschrift, die ins Corpus Inscriptionum Latinarum (CIL) XIII unter der Nummer 4144 aufgenommen, aber von KARL ZANGEMEISTER nicht selbst in Augenschein genommen wurde bzw. werden konnte (s.u.). Im CIL ist folgende Lesung abgedruckt:

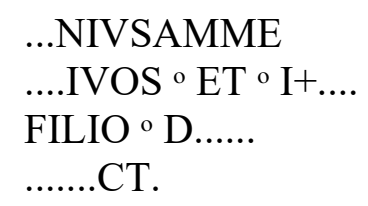

Beim Buchstabenrest am Ende von Zeile 2 handelt es sich laut CIL um die linke Schräge des „, $\mathrm{V}^{\prime}$. Es liegt somit die Grabinschrift eines Mannes vor, der [---]nius Amme[---] hieß. Er hat sie [sibi v]ivos ${ }^{32}$ (für sich zu Lebzeiten) und für Iu[---], filio (Iu[---], seinen Sohn), der bereits verstorben war, erstellen lassen. Dem überlieferten Text könnte ein $D($ is) M(anibus) vorangegangen sein. Im unteren Bereich könnte ebenfalls Text fehlen. Der Stein dürfte ins ausgehende 2. Jahrhundert oder in die 1. Hälfte des 3. Jahrhunderts datieren.

Erstmals mitgeteilt wird der Fund von XAVER Boos im Jahre 1821. Er gibt an, die Inschrift bei der weitgehend zerstörten (nach der Mutter Karls des Großen benannten) Bertrada-Burg ${ }^{33}$ bei Mürlenbach gesehen zu haben: „Rechts am Eingang des noch bewohnbaren Stück Gebäudes, das dem Oberförster oder Garde à cheval, wie man damals solche Forstbediente nannte, zur Wohnung diente, entdeckte ich in einer Höhe von 10-12 Schuh einen Theil einer römischen Inschrift, die ich also las:

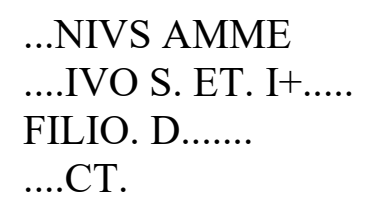

Vielleicht [lautete] die ganze, von dem Hammer des Maurers noch nicht beschädigte, Inschrift dieses eingemauerten römischen Denkmals: Junius Ammerinus vivo sibi et Junio filio defuncto fecit oder fieri curavit.“

Dass der Ergänzungsvorschlag nicht völlig aus der Luft gegriffen ist, zeigt die Erwähnung desselben in der weiteren Forschung des 19. Jahrhunderts. ${ }^{34}$ Insbesondere ein dem Wort filio folgendes d[efuncto] in ausgeschriebener oder abgekürzter Form

\footnotetext{
${ }^{31}$ Siehe zu dem mutmaßlichen Quellheiligtum Trierer Zeitschrift 24-26, 1956-58, 561f.; KRAUSSE 2006, 165 Nr. 1207.

${ }^{32}$ BRAMBACH 1867, 170 Nr. 848 liest in Zeile 2 [v]ivo s[ibi] et Iu[---]. Die Wendung vivo sibi ist im gallisch-germanischen Raum aber so gut wie unbekannt, während es für die Schreibweise sibi vivos (statt vivus) sehr viele Beispiele gibt. Unwahrscheinlich ist die bei WEILER/REINARD 2018, 194, vertretene Sicht, in [---]ivos das Ende eines Namens erkennen zu wollen.

${ }^{33}$ In der Literatur findet sich immer wieder der Hinweis, dass die Burg wahrscheinlich auf den Überresten eines römischen Kastells erbaut worden sei; siehe SCHNEIDER 1843, 83; BÄRSCH 1854, 291; MÜLLER 1932, 43. Hierfür fehlt es aber an Belegen; siehe LOSSE 2000, 132.

${ }^{34}$ Siehe BÄRSCH 1825, 893f.; BÄRSCH 1854, 291; BRAMBACH 1867, 170 Nr. 848 (sicherlich versehentlich „Ammesinus“ statt Ammerinus).
} 
beansprucht einige Wahrscheinlichkeit. ${ }^{35}$ Im Zuge verschiedener Umbauarbeiten und Restaurierungen in und an der erwähnten Burg ist die Inschrift früh verschollen. GEORG BÄRSCH teilt schon 1825 mit, dass sie nicht mehr auffindbar sei. ${ }^{36}$ Alle späteren Bearbeiter der Inschrift mussten also von der bei Boos abgedruckten Lesung ausgehen.

Bereits im 19. Jahrhundert ist man aber zum Teil dazu übergegangen, die von Boos als Vorschlag formulierte Ergänzung als tatsächlich von ihm gelesene Inschrift wiederzugeben. ${ }^{37}$ Der oben erwähnte MüLLER schreibt ebenfalls: „Innerhalb der Burg war an einem Turme folgende römische Inschrift angebracht", um dann, ohne jeden Nachweis, die Ergänzung von Boos wiederzugeben. ${ }^{38}$ Zuletzt ist 2018 von JOSEPH GROBEN in seinem Buch zum Kylltal ausgeführt worden: „Eine schöne Grabinschrift, die auf einem eingemauerten Stein gefunden wurde, bezeugt, dass Junius Ammerinus seinem verstorbenen Sohn ein Denkmal gesetzt hat" mit der Lesung „IVNIVS AMMERINVS / VIVO SIBI ET IVNIO FILIO DEFVNCTO / FECIT“ und der anschließenden Vermutung: „Solche persönlichen Zeugnisse von ,Pietät‘ berühren vielleicht noch stärker als Steine und Münzen. Fast greifbar treten einzelne Individuen aus dem Dunkel der Vergangenheit hervor. “39 Die Aufarbeitung der Überlieferungsgeschichte dieses Inschriftfragments zeigt, dass diese Schlussfolgerung nicht haltbar ist.

Selbst wenn uns die genauen Namen in dieser Grabinschrift letztlich verborgen bleiben: In Mürlenbach und der näheren Umgebung, so erweisen neben den archäologischen Befunden die hier vorgestellten Inschriften, hat sich ein römische Erbe erhalten, das einmal mehr zeigt, wie dicht die römische Eifel besiedelt war. ${ }^{40}$

\footnotetext{
${ }^{35}$ Angesichts der Länge der vorangehenden Zeilen und dem, soweit ersichtlich, Umstand, dass Wortende und Zeilenende offenbar zusammenfallen, ist die Lesung $d[e f u n] c t[o]$ weniger wahrscheinlich.

${ }^{36}$ BÄRSCH 1825, 894 Anm. *: „Das bezeichnete Gebäude dient jetzt zum Schaafstall. Ich habe dasselbe genau untersucht, den beschriebenen Stein aber nicht mehr vorgefunden, welches auch nicht zu verwundern ist, indem mit dem Gebäude bedeutende Veränderungen vorgenommen worden. "Skeptischer klingt dies bei BÄRSCH 1854, 291: „Boos behauptete, am Eingange des vormals von dem Förster bewohnten Gebäudes auf der Burg einen Stein mit der Inschrift gefunden zu haben [es folgt eine ungenauere Wiedergabe des Textes als in 1825]. Im Jahre 1824 diente das bezeichnete Gebäude zum Schaafstalle, ich untersuchte dasselbe damals genau, konnte aber den Stein nicht auffinden." Ebenfalls zweifelhaft ist für BÄRSCH 1825, 894 die Mitteilung bei MiNOLA ${ }^{2}$ 1816, 309, dass in Mürlenbach ,in der zweyten Hälfte des XVIII. Jahrhunderts Hirtenknaben, welche einer Maus nachjagten, auf verborgenes Gold- und Silbergeräth stiessen; die Aufschriften waren Römisch, und von Nero.“ Hierzu BÄRSCH 1825, 894: ,[...] in Mürlenbach selbst habe ich nichts darüber ausmitteln können.“

${ }^{37}$ Soweit hier bekannt erstmals bei SCHNEIDER 1843, 83, der mit Hinweis auf Boos bemerkt: „Vor mehren [sic] Jahren sah man noch einen eingemauerten Stein mit folgender zum Theil verlöschten Inschrift“, und im Anschluss lediglich den Ergänzungsvorschlag abdruckt.

${ }^{38}$ MÜLLER 1932, 43.

${ }^{39}$ GROBEN 2018, 185 mit Hinweis auf Boos 1821 und BECKER 2016. Bei letzterem wird die Grabinschrift freilich gar nicht erwähnt.

${ }^{40} \mathrm{Zu}$ den Heiligtümern entlang der römischen Fernstraße zwischen Trier und Köln erscheint vom Verfasser demnächst der Artikel „Von Trier über Bitburg Richtung Köln: Gallo-römische Heiligtümer an der Via Agrippa“.
} 


\section{Literaturverzeichnis}

AUS'M WeERTh 1876 = E. AUS'M WeERTh, Der Juno-Tempel bei Nattenheim, BJ 57, $1876,56-65$.

BÄRSCH 1825 = G. BÄRSCH, Eiflia Illustrata I 2, Aachen/Leipzig 1825.

BÄRSCH 1854 = G. BÄRSCH, Eiflia Illustrata III 2,1, Aachen/Leipzig 1854.

BECKER 2016 = E. BECKER, Spuren der Kelten und Römer in und um Mürlenbach, Landkreis Vulkaneifel - Heimatjahrbuch 2016, $194 \mathrm{f}$.

BINSFELD u.a. 1988 = W. BINSFELD/K. GOETHERT-PolASCHEK/L. SCHWIndEN, Kata$\log$ der römischen Steindenkmäler des Rheinischen Landesmuseums Trier 1: Götter- und Weihedenkmäler, Trierer Grabungen und Forschungen XII 1, CSIR Deutschland IV 3: Gallia Belgica: Trier und Trierer Land, Mainz 1988.

Boos 1821 = X. Boos, Bertrada von Mürlenbach, Trierische Kronik 6, 1821, 76-81.

BRAMBACH 1867 = W. BRAMBACH, Corpus inscriptionum Rhenanarum, Elberfeld 1867.

CABUY $1991=$ Y. CABUY, Les temples gallo-romains des cités des Tongres et de Trévires, Publications Amphora 12, Brüssel 1991.

FLORENCOURT $1842=$ W. CHASSOT VON FLORENCOURT, Beiträge zur Kunde alter Götterverehrung, Trier 1842.

GHETTA $2008=$ M. GHETTA, Spätantikes Heidentum. Trier und das Trevererland, Trier 2008.

GRENIER $1960=$ A. GRENIER, Manuel d'archéologie gallo-romaine 4,2: Les monuments des eaux. Villes d'eau et sanctuaires de l'eau, Paris 1960.

Groben 2018 = J. Groben, Das stille Tal der Kyll. Geschichte, Dichtung, Malerei, Musik, Trier 2018.

HAGEN ${ }^{2} 1931=$ J. HAGEN, Die Römerstrassen der Rheinprovinz, Erläuterungen zum Geschichtlichen Atlas der Rheinprovinz 8, Publikationen der Gesellschaft für Rheinische Geschichtskunde 12,8, 2., überarb. Aufl., Bonn 1931.

HENRICH $2006=$ P. HENRICH, Die römische Besiedlung in der westlichen Vulkaneifel, Beiheft Trierer Zeitschrift 30, Trier 2006.

HetTNER 1893 = F. HetTNer, Die römischen Steindenkmäler des Provinzialmuseums zu Trier mit Ausschluss der Neumagener Monumente, Trier 1893.

HETTNER 1911 = F. HETTNER, Nachtrag zu dem Werke: „Drei Tempelbezirke im Trevererlande“, Trierer Jahresberichte, N.F. 3, 1910 (1911), 49-67.

Horne/King $1980=$ P. D. Horne/A. C. KInG, Romano-Celtic Temples in Continental Europe: A Gazetteer of those with Known Plans. In: W. Rodwell (Hg.), Temples, Churches and Religion: Recent Research in Roman Britain with a Gazetteer of Romano-Celtic Temples in Continental Europe. Part 1, BAR British Series 77, Oxford 1980, 369-555.

KaKoschKe $2010=$ A. KAKoschKe, Die Personennamen in der römischen Provinz Gallia Belgica, Alpha-Omega Reihe A: Lexika, Indizes, Konkordanzen zur klassischen Philologie 255, Hildesheim/Zürich/New York 2010. 
KRAUSSE 2006 = D. KRAUSSE, Eisenzeitlicher Kulturwandel und Romanisierung im Mosel-Eifel-Raum. Die keltisch-römische Siedlung von Wallendorf und ihr archäologisches Umfeld. Fundstellenkatalog, unter Mitarbeit von A. FisCHBOCK, Römisch-Germanische Forschungen 63, Mainz 2006.

LOBÜSCHER $1997=$ TH. LOBÜSCHER, Deus Caprio: eine verschollene Weihung aus Mürlenbach (Kr. Daun), Archäologisches Korrespondenzblatt 27, 1997, 159161.

LOSSE $2000=$ M. LossE, Bertradaburg Mürlenbach, Landkreis Vulkaneifel - Heimatjahrbuch 2000, 132-141.

MinOLA ${ }^{2} 1816=$ A. B. MinOlA, Kurze Uebersicht dessen, was sich unter den Römern seit Jul. Cäsar bis auf die Eroberung Galliens durch die Franken am Rheinstrome Merkwürdiges ereignete, 2., verm. und verb. Aufl., Köln 1816.

MüLLER 1932 = J. H. MüLLER, Heimatgeschichte von Mürlenbach. Aus dem Land der Karösen, Mürlenbach 1932.

SCHNEIDER 1843 = J. SCHNEIDER, Das Kyllthal mit seinen nächsten Umgebungen, nach geschichtlich-antiquarischem und naturhistorischem Bezug und mit Rücksicht auf die Sagen, Trier 1843.

WACKENRODER 1927 = E. WACKENRODER (Bearb.), Die Kunstdenkmäler des Kreises Prüm, Die Kunstdenkmäler der Rheinprovinz 12/2, Düsseldorf 1927.

WEILER/REINHARD $2018=$ S. WEILER/P. REINARD, Inschriften aus Bitburg und der südlichen Eifel aus der Römerzeit (I.BiER) - Katalog und Auswertung, Bitburg 2018 .

\section{Kontakt zum Autor:}

Krešimir Matijević

Europa-Universität Flensburg

E-Mail: Kresimir.Matijevic@,uni-flensburg.de

\section{(cc) EY}

Dieser Beitrag ist lizenziert unter einer Creative Commons Namensnennung - 4.0 International Lizenz. 